\title{
Relationship between Static Anterior Laxity Using the KT-1000 and Dynamic Tibial Rotation during Motion in Patients with Anatomical Anterior Cruciate Ligament Reconstruction
}

\author{
KOJI SATO, AKIRA MAEDA*, YOSHIO TAKANO**, HIROO MATSUSE ${ }^{\dagger}$, \\ HIROFUMI IDA ${ }^{\ddagger}$ AND NAOTO SHIBA ${ }^{\S}$

\begin{abstract}
Kurume University Graduate School of Medicine, ${ }^{\prime}$ Division of Rehabilitation, Kurume University Hospital,
${ }^{\S}$ Department of Orthopaedic Surgery, Kurume University School of Medicine, Kurume 830-0011,

*Narita Orthopaedic Hospital, Fukuoka 812-0018, **Department of Physical Therapy,

International University of Health and Welfare School of Health Sciences, Okawa 137-1,

${ }^{*}$ Department of Sports Management, Jobu University, Isesaki 372-8588, Japan
\end{abstract} \\ Received 16 November 2012, accepted 7 February 2013 \\ J-STAGE advance publication 7 August 2013 \\ Edited by KOICHI YAMAKI
}

\begin{abstract}
Summary: The anterior cruciate ligament (ACL) plays an important role in controlling knee joint stability, not only by limiting tibial anterior translation but also by controlling knee axial rotation. The aim of ACL reconstruction is to reduce excessive anterior joint laxity, hoping to restore normal tibiofemoral kinematics including knee axial rotation. The purpose of this study was to investigate the relationship between static anterior instability and tibial rotation during several activities in an anterior cruciate ligament reconstructed knee. Seven patients with unilateral ACL injury performed plain walking, running, landing and side step cutting tasks after ACL reconstruction with a mean follow-up of 14 months. The kinematic data for the 4 motions was measured using a motion analysis system and the point cluster technique. The evaluation period was defined to be from the first contact to removal of the tested leg from the ground. Maximum tibial internal rotation during tasks was calculated using the point cluster technique (PCT). Passive anterior tibial translation was measured using a KT-1000 arthrometer. Regression analysis was used to determine the correlation of the maximum internal rotation with the side-to-side difference of static anterior tibial translation measured using a KT-1000 arthrometer. During side step cutting maneuvers, maximum tibial internal rotation significantly showed negative correlation with static anterior tibial translation $(\mathrm{p}<0.05, \mathrm{r}=0.83)$. The anterior laxity contributed to the normal knee rotation kinematics. The normal anterior tibial translation obtained by ACL reconstruction is thought to be the key factor in successful restoration of normal knee kinematics.
\end{abstract}

Key words anterior cruciate ligament, kinematics, tibial rotation, anterior tibial translation

\section{INTRODUCTION}

Recently, anatomical anterior cruciate ligament (ACL) reconstruction has been widely used to restore not only anterior tibial instability but also rotational stability. In vivo study has reported that anatomical ACL reconstruction provided better control of dynamic rotatory stability than single-bundle reconstruction [1].

The anterior laxity of the knee joint is an important parameter for evaluating a knee with ACL insufficiency. To obtain objective evaluation methods, noninvasive arthrometer such as the KT-1000 were developed [2]. This method has become the standard method for clinically evaluating anterior knee laxity before 
and after ACL reconstruction. Previous studies reported a positive correlation between passive anterior knee laxity measured using a KT-1000 arthrometer and anterior tibial translation during dynamic activity using bi-plane fluoroscopy [3]. These findings indicate that static anterior knee laxity is associated with anterior tibial translation during dynamic activities. Although many studies have observed knee kinematics, there are few studies on the correlation between passive anterior tibial laxity and axial tibial rotation during walking or sports activities.

The purpose of this study was to determine the relationship between anterior knee laxity measured using a KT-1000 arthrometer and axial rotation during 4 tasks including hi-demand activity [(1) plain walking, (2) running, (3) landing from a $30 \mathrm{~cm}$ high platform on both feet, (4) side step cutting].

\section{MATERIALS AND METHODS}

Subjects were patients who received ACL reconstruction at our hospital between 2010 and 2011. Exclusion criteria were any prior significant injury to the contralateral knee as well as significant damage to other knee structures in the ACL reconstructed knee (subjects with minor meniscal tears, requiring removal of no more than one third of the radial width of the meniscus, were not excluded). All patients who were not excluded by these criteria and who underwent ACL reconstruction were asked to participate in the study. Informed consent was obtained from seven subjects. Seven subjects with unilateral ACL reconstructions participated in this study ( 3 male and 4 female; mean age $21.3 \pm 4.9$ years). The time interval between operation and data collection was more than 9 months for all subjects (mean: 14.3 \pm 1.8 month). All subjects provided written informed consent in compliance with Kurume University Ethics Committee guidelines.

\section{Surgical technique and rehabilitation}

In all patients, anatomical double-bundle ACL reconstructions were performed by the same physician. The injured knee was put on the operating table, with a foot rest and with lateral thigh support to obtain 90 degrees of flexion. The hamstring tendons were harvested over pes anserinous. Diagnostic arthroscopy was performed through the anterolateral and anteromedial portals. After confirming the rupture of the anteromedial (AM) and posterolateral (PL) bundles, two femoral and two tibial tunnels were created to reproduce both the AM and PL bundle anatomically using the hamstring tendon graft. After the PL graft was secured in the femoral tunnel by use of an EndoButton CL (Smith \& Nephew Endoscopy), the AM graft was then secured in the same manner. Then, both the PL bundle and AM bundle grafts were fixed using a Double Spike Plate (DSP, Smith \& Nephew Endoscopy) under tension of $20 \mathrm{~N}$ at 20 degrees of knee flexion.

All patients received the same ACL rehabilitation protocol. After two weeks of immobilization range of motion exercises were started. Partial weight bearing was allowed at two weeks, and full weight bearing was started at 3 weeks. Jogging was allowed at 3 months, and return to previous sports activities was permitted at 9 to 10 months, depending on the recovery of the patients' quadriceps muscle strength and other conditions.

\section{Experimental procedure}

Before data collection, clinical evaluations were performed in all subjects. We obtained Tegner, Lysholm and International Knee Documentation Committee (IKDC) scores. In addition, we evaluated passive anterior tibial translation using the KT-1000 arthrometer (MEDmetric Corp, San Diego, CA) for both the reconstructed and contralateral knees. The measurements were performed using manual maximum force at the tibia. Side to side differences between the reconstructed

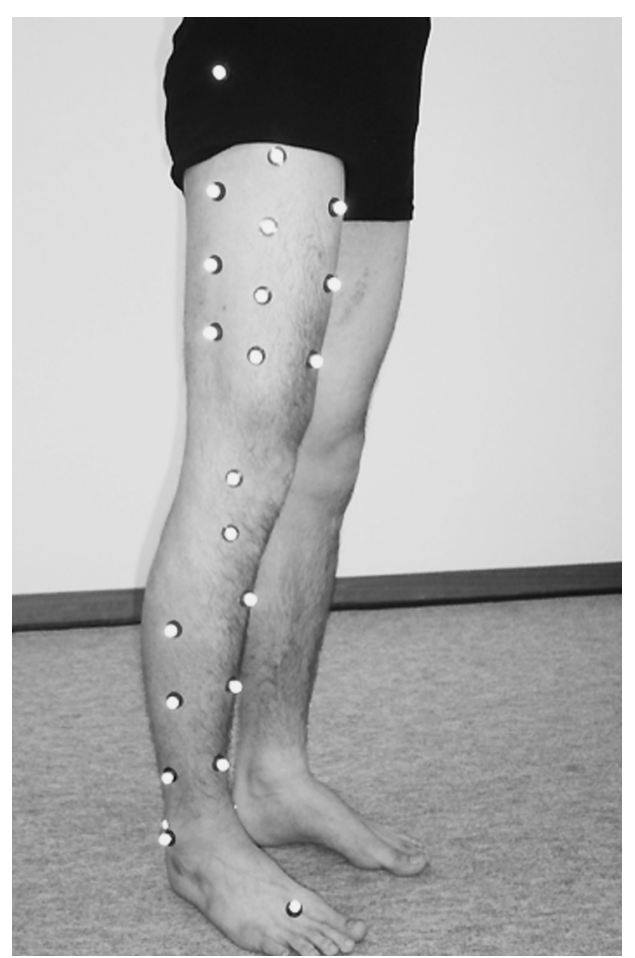

Twenty five $9 \mathrm{~mm}$ reflective markers were attached to the limb.

Fig. 1. Arrangement of markers for the point cluster technique. 
knee and the contralateral knee were calculated.

An optical motion analysis system with 9 cameras (Vicon612, Vicon Motion Systems Ltd, Oxford, United Kingdom) was used to record the 3 dimensional movements of lower extremities at a sampling frequency of $200 \mathrm{~Hz}$. The ground reaction force was measured using a force plate (AMTI, MA, USA) embedded in the floor. The vertical ground reaction force was used to signal the initial contact to determine the data capture period. A total of twenty five reflective markers $9 \mathrm{~mm}$ in diameter were attached to the lower limb using double-sided adhesive tape (Fig. 1). The markers were used to implement the point cluster method. The point cluster technique (PCT), developed by Andriacchi and coworkers, is a method designed to derive limb segment motion from markers placed on the skin(4). Our algorithm for the PCT was developed following the procedure described by Andriacchi et al. [4]. For the PCT, the skin markers were divided into two groups: For a cluster of points, ten and six markers were attached on the thigh and shank respectively. The body landmarks were attached to the greater trochanter, the lateral and medial epicondyles of the femur, the lateral and medial edges of the tibial plateau, the lateral (fibula) and medial malleoli, the fifth metatarsophalangeal joint, the first distal phalanx head, and the heel. The subjects were first measured in a static standing position to define the initial reference position of the axes.

\section{Experimental tasks}

All subjects were barefoot and were given enough time to warm up to familiarize themselves with the 4 tasks. They were asked to perform 4 different activities on both sides : (1) plain walking, (2) running, (3) landing from $30 \mathrm{~cm}$ high platform on both feet, (4) side step cutting. They performed 3 trials of the each tasks.
As a control group, we measured the contralateral intact knee for comparison with the reconstructed knee.

\section{Kinematic data collection}

The evaluation period was defined as from the first contact to the removal of the tested leg from the floor. Foot contact was determined by the force plate when vertical ground reaction force was presented. Using the PCT for each trial, the angular displacement of tibial rotation was calculated according to the joint coordinate system. For comparison, we calculated the average of three trials of each tasks to compare the reconstructed knee with the contralateral knee.

\section{Statistical analysis}

Statistical analysis was performed with JMP10 software (SAS Institute Inc.). Correlations between the maximum tibial internal rotation during tasks and the static anterior tibial translation (KT-1000 arthrometer scores) were expressed in terms of the Spearman rank correlation coefficient. The level of significance was set at $\mathrm{p}<0.05$.

\section{RESULTS}

A summary of subject characteristics is shown in Table 1. All patients resumed their preinjury level of sports participation. The median Lysholm score was 96 (range, 94-100) and median Tegner score was 7 (range 7-9) after surgery. The median IKDC subjective score was 88 (range 82.8-100) (Table 1). Means and standard deviations for all kinematic data in four tasks are presented in Table 2.

The correlation between maximum internal rotation and the KT-1000 arthrometer score in side step cutting was shown in Fig. 2. During side step cutting,

TABLE 1.

Patient Demographics and Information Including Tegner Score, Lysholm Score and KT-1000 side-to-side Differences (mm)

\begin{tabular}{cccccccccc}
\hline ID & Sex & Age & $\begin{array}{c}\text { Height } \\
(\mathrm{cm})\end{array}$ & $\begin{array}{c}\text { Weight } \\
(\mathrm{kg})\end{array}$ & $\begin{array}{c}\text { Months since } \\
\text { surgery }\end{array}$ & $\begin{array}{c}\text { Tegner } \\
\text { score }\end{array}$ & $\begin{array}{c}\text { Lysholm } \\
\text { score }\end{array}$ & IKDC & KT-1000 \\
\hline 1 & F & 18 & 163 & 61 & 13 & 7 & - & - & -2 \\
2 & F & 18 & 162 & 59 & 14 & 7 & - & - & -2 \\
3 & M & 20 & 175 & 66 & 15 & 9 & 94 & 82.8 & 0 \\
4 & M & 21 & 172 & 61 & 18 & 7 & 99 & 96.6 & 5 \\
5 & F & 29 & 161 & 52 & 13 & 7 & 95 & 68.7 & 1 \\
6 & M & 16 & 173 & 54 & 14 & 5 & 100 & 100 & 5 \\
7 & F & 27 & 155 & 60 & 13 & 7 & 95 & 91.9 & 0 \\
mean & & 21.3 & 165.9 & 59.0 & 14.3 & 7.0 & 96.6 & 88.0 & 1.4 \\
SD & & 4.9 & 7.5 & 4.7 & 1.8 & 1.2 & 2.7 & 12.6 & 2.6 \\
\hline
\end{tabular}


TABLE 2.

Maximum Internal Rotation Angle in Each Motion (deg)

\begin{tabular}{|c|c|c|c|c|c|c|c|c|}
\hline \multirow{2}{*}{ ID } & \multicolumn{2}{|c|}{ walking } & \multicolumn{2}{|c|}{ running } & \multicolumn{2}{|c|}{ landing } & \multicolumn{2}{|c|}{ side step cutting } \\
\hline & reconstruct & contralateral & reconstruct & contralateral & reconstruct & contralateral & reconstruct & contralatera \\
\hline 1 & 11.1 & 13.2 & 0.7 & 4.5 & 8.7 & 5.9 & 9.9 & 7.4 \\
\hline 2 & 1.8 & 7.4 & 1.9 & 9.7 & 5.3 & 11.7 & 4.2 & 12.6 \\
\hline 3 & 0.4 & 8.9 & 8.6 & 18.5 & 18.1 & 17.9 & 10.5 & 17.7 \\
\hline 4 & -1.1 & 10.7 & 2.3 & 7.1 & 9.5 & 9.5 & 6.4 & 9.8 \\
\hline 5 & 6.1 & 11.6 & 3.2 & 9.0 & 11.5 & 10.7 & 10.8 & 15.2 \\
\hline 6 & -0.5 & -1.7 & 3.1 & 1.0 & 4.6 & 17.4 & 3.0 & 8.7 \\
\hline 7 & 4.9 & 8.3 & 6.2 & 13.1 & 14.6 & 13.6 & 10.7 & 17.7 \\
\hline mean & 3.3 & 8.4 & 3.7 & 9.0 & 10.3 & 12.4 & 7.9 & 12.7 \\
\hline $\mathrm{SD}$ & 4.4 & 4.9 & 2.7 & 5.7 & 4.9 & 4.3 & 3.4 & 4.3 \\
\hline
\end{tabular}

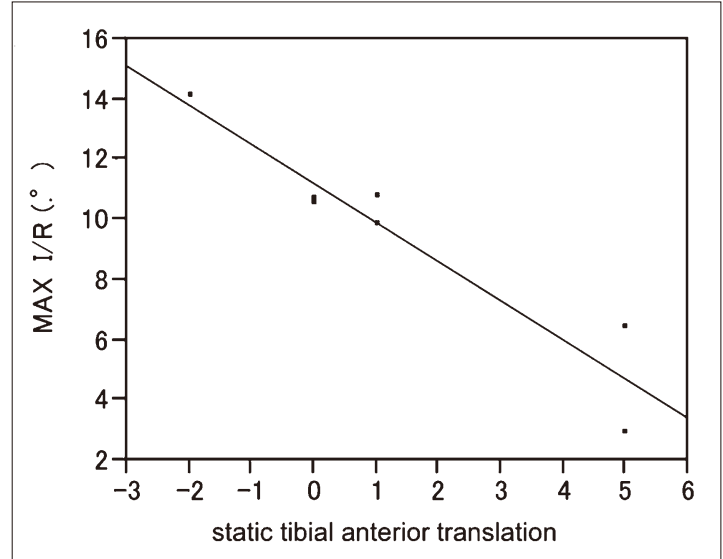

Liner regression plot for maximum internal rotation during side step cutting to side-to-side difference of static anterior tibial translation using KT-1000

Fig. 2. Side step cutting.

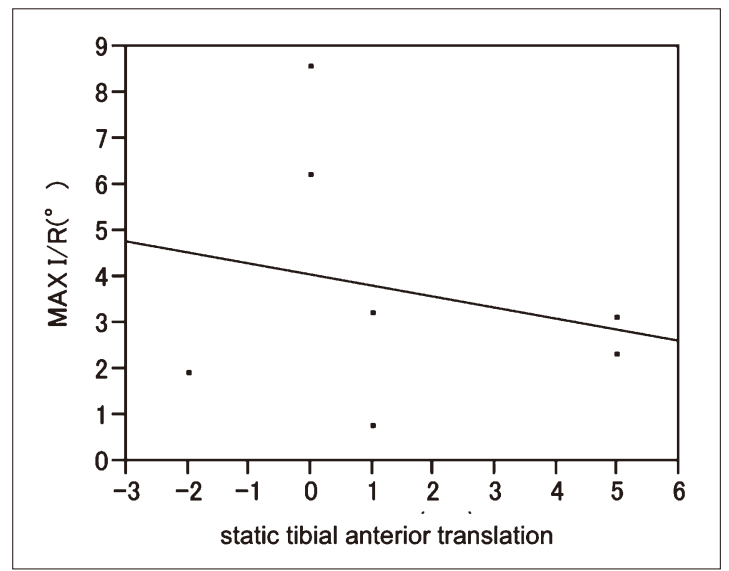

Liner regression plot for maximum internal rotation during running to side-to-side difference of static anterior tibial translation using KT-1000.

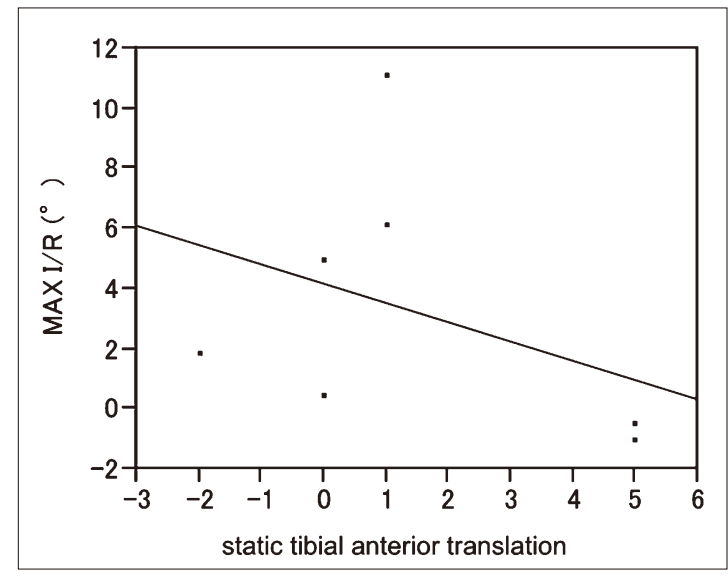

Liner regression plot for maximum internal rotation during walking to side-to-side difference of static anterior tibial translation using KT-1000

Fig. 3. Plain walking

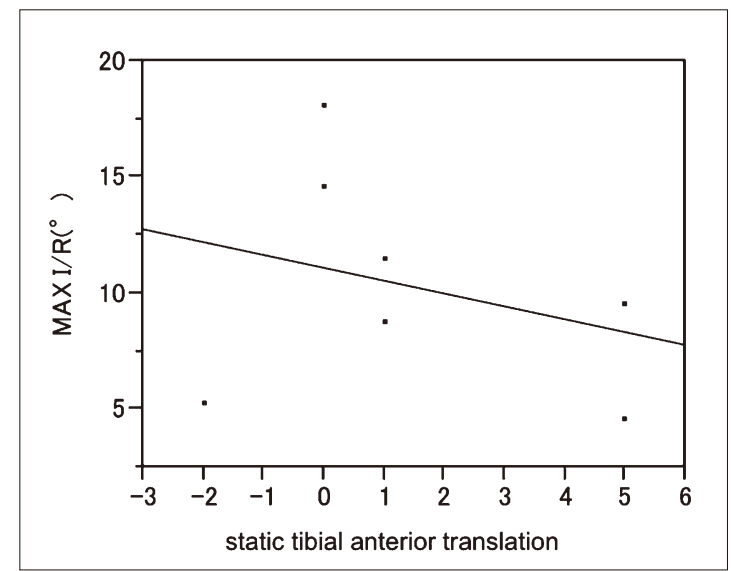

Liner regression plot for maximum internal rotation during landing to side-to-side difference of static anterior tibial translation using KT-1000.

Fig. 4. Running.

Fig. 5. Landing. 
there was a significant negative correlation between the maximum internal rotation and the side-to-side difference of static anterior tibial translation $(\mathrm{p}=0.0220$, $\mathrm{r}=0.83$ ). There was no significant correlation of the maximum internal rotation to the KT-1000 arthrometer score in plain walking, running and landing from a platform (Fig. 3-5).

\section{DISCUSSION}

In this study, during low demand activities such as walking, running and landing, there were no significant correlations between static anterior tibial laxity measured with KT-1000 arthrometer and maximum internal tibial rotation. However, during more stressful activity such as side step cutting maneuvers, there was significant negative correlation between static anterior tibial translation and maximum internal rotation.

Although a previous study found that tibial rotation could be restored to the normal level after an ACL reconstruction during low-demand activities such as walking [5], stressful activity may be followed by subsequent pivoting to create rotational loads on the knee. The application of such loads on the knee could provide additional insights into functional recovery after an ACL reconstruction [6]. The authors of that study concluded that under high-stress activities, ACL reconstruction may not restore tibial rotation to the previous physiological level, even though anterior tibial translation is restored.

Torry et al. reported a strong relationship between passive anterior knee laxity measured by KT-1000 and peak anterior tibial translation during dynamic activity in otherwise healthy persons performing a stiff droplanding motion [3]. In this study, we demonstrated a negative correlation between passive anterior tibial laxity by KT-1000 arthrometer and maximum internal tibial rotation.

Carpenter et al. demonstrated that when knees were fully extended, tibiae in ACL reconstructed knees were externally rotated compared with contralateral knees [7]. The result of this study showed that the negative anterior knee laxity contributed to the normal knee rotation kinematics. On the other hand, increasing anterior knee laxity contributed to excessive external rotation. We suggested that the subjects with positive anterior knee laxity performed compensatory motion to reduce tibial internal rotation. The external tibial rotation shifted the tibial contact area primarily in the lateral compartment. Thus, these movement pattern shifts could significantly alter the location, pattern, and magnitude of stress applied to both cartilage and menisci [8].

This study demonstrated that static anterior knee laxity measured by KT-1000 arthrometer is associated with tibial rotation during high demand activities. The normal anterior tibial translation obtained by ACL reconstruction is thought to be the key factor in restoring the normal knee kinematics.

Previous study demonstrated of the kinematics of high demand activities found no significant differences between the knees in the control group and the intact knees in the reconstruction group [9]. Therefore we decided to use the intact contralateral knee in the reconstruction group as a normal control.

A limitation of this study was the number of participants included in the short-term follow-up, and the relative inaccuracy of the gait analysis systems. The sample size and statistical power may be insufficient to detect the relationships we reported.

Future studies will include a larger sample size and a comparison with intact knee kinematics. There was variability in patient data in this study. We need to be able to better compare data between patients. The reference of the skin based marker motion analysis system was based on a static standing position. This made it difficult to determine the actual initial joint angle, especially the axial rotation angle. Future research is required to determine the actual initial joint angle for kinematic analysis.

\section{REFERENCES}

1. Yagi M, Kuroda R, Nagamune K, Yoshiya S, and Kurosaka M. Double-bundle ACL reconstruction can improve rotational stability. Clin Orthop Relat Res 2007; 454:100-107.

2. Isberg J, Faxen E, Brandsson S, Eriksson BI, Karrholm J et al. KT-1000 records smaller side-to-side differences than radiostereometric analysis before and after an ACL reconstruction. Knee Surg Sports Traumatol Arthrosc 2006; 14(6):529-535.

3. Torry M, Myers C, Pennington W, Shelburne KB, Krong JP et al. Relationship of anterior knee laxity to knee translations during drop landings: a bi-plane fluoroscopy study. Knee Surg Sports Traumatol Arthrosc 2011; 19:653-662.

4. Andriacchi TP, Alexander EJ, Toney MK, Dyrby C, and Sum J. A point cluster method for in vivo motion analysis: applied to a study of knee kinematics. J Biomech Eng 1998; 120(6):743-749.

5. Georgoulis AD, Papadonikolakis A, Papageorgiou CD, Mitsou A, and Stergiou N. Three-dimensional tibiofemoral kinematics of the anterior cruciate ligament-deficient and reconstructed knee during walking. Am J Sports Med 2003; 31:75-79.

6. Ristanis S, Stergiou N, Patras K, Vasiliadis HS, Giakas G et al. Excessive tibial rotation during high-demand activities is not restored by anterior cruciate ligament recon- 
struction. Arthroscopy 2005; 21:1323-1329.

7. Carpenter RD, Majumdar S, and Ma CB. Magnetic resonance imaging of 3-dimensional In vivo tibiofemoral kinematics in anterior cruciate ligament-reconstructed knees. Arthroscopy 2009; 25:760-766.

8. Tashman S, Collon D, Anderson K, Kolowich P, and Anderst W. Abnormal rotational knee motion during run- ning after anterior cruciate ligament reconstruction. Am J Sports Med 2004; 32:975-983.

9. Misonoo G, Kanamori A, Ida H, Miyakawa S, and Ochiai N. Evaluation of tibial rotational stability of single-bundle vs. anatomical double-bundle anterior cruciate ligament reconstruction during a high-demand activity - A quasirandomized trial. The Knee 2012; 19:87-93. 